\title{
Genetic characterization of avian influenza subtype H4N6 and H4N9 from live bird market, Thailand
}

\author{
Trong Wisedchanwet ${ }^{1}$, Manoosak Wongphatcharachai ${ }^{2}$, Supanat Boonyapisitsopa ${ }^{1}$, Napawan Bunpapong ${ }^{1}$, \\ Pravina Kitikoon ${ }^{3}$, Alongkorn Amonsin 1,2* $^{*}$
}

\begin{abstract}
A one year active surveillance program for influenza A viruses among avian species in a live-bird market (LBM) in Bangkok, Thailand was conducted in 2009. Out of 970 samples collected, influenza A virus subtypes H4N6 $(n=2)$ and H4N9 $(n=1)$ were isolated from healthy Muscovy ducks. All three viruses were characterized by whole genome sequencing with subsequent phylogenetic analysis and genetic comparison. Phylogenetic analysis of all eight viral genes showed that the viruses clustered in the Eurasian lineage of influenza A viruses. Genetic analysis showed that H4N6 and H4N9 viruses display low pathogenic avian influenza characteristics. The HA cleavage site and receptor binding sites were conserved and resembled to LPAI viruses. This study is the first to report isolation of H4N6 and H4N9 viruses from birds in LBM in Thailand and shows the genetic diversity of the viruses circulating in the LBM. In addition, co-infection of H4N6 and H4N9 in the same Muscovy duck was observed.
\end{abstract}

\section{Findings}

Live-bird markets (LBMs) are the places where wild birds, pet birds, meat birds and domestic poultry are sold to households. In Asia including Thailand, due to the cultural preference of consuming freshly slaughtered poultry, LBMs are located in both suburban areas and center of the communities. In the markets, thousands of birds from different sources are sold in wire stacked cages containing densely packed and mixed bird populations. These conditions provide excellent environments for animal to animal and animal to human influenza virus transmissions and may result in an outbreak of influenza A virus in both animals [1,2] and humans $[3,4]$. Therefore, LBMs are considered a major source of influenza A virus dissemination and potential influenza A virus reassortment $[5,6]$.

Up to date, many studies on influenza A in LBMs from various countries have been reported. During 2000-2001, 6 subtypes (9 genotypes) of low-pathogenic avian influenza (LPAI) were identified in LBMs in

\footnotetext{
* Correspondence: alongkorn.A@chula.ac.th

'Emerging and Re-emerging Infectious Diseases in Animals, Research Unit, Faculty of Veterinary Science, Chulalongkorn University, Bangkok, Thailand Full list of author information is available at the end of the article
}

China [7]. Apart from Asian countries, in the US, H5N2 low pathogenic avian influenza (LPAI) viruses have been isolated from LBMs in several states in the $80 \mathrm{~s}$ [8]. In Thailand, only one study of influenza A viruses recovered from LBMs has ever been reported [9]. In that study, highly pathogenic avian influenza (HPAI) H5N1 viruses were isolated from both bird carcasses and healthy birds during the 2006-2007 LBM and local food market (LFM) surveillance program. The findings suggested that animal movement from H5N1 outbreak areas may introduce the virus into the markets and play an important role in emergence or re-emergence of influenza A in animals in Thailand [9]. Since LBMs play an important role in the dissemination of avian influenza virus, active surveillance of influenza A virus in LBMs is important in order to develop an early warning system and implement prevention and control strategies for influenza A outbreaks. In this study, a one year active surveillance program for influenza A viruses among avian species in LBM from Bangkok, Thailand was conducted in 2009. Influenza A subtypes, H4N6 $(n=2)$ and H4N9 $(n=1)$, were isolated from healthy Muscovy ducks. Interestingly, co-infection of H4N6 and H4N9 in the same Muscovy duck was also observed.

C Biomed Central

(C) 2011 Wisedchanwet et al; licensee BioMed Central Ltd. This is an Open Access article distributed under the terms of the Creative Commons Attribution License (http://creativecommons.org/licenses/by/2.0), which permits unrestricted use, distribution, and reproduction in any medium, provided the original work is properly cited. 
Whole genome sequencing, phylogenetic analysis and genetic analysis of the viruses were performed. This study highlights the first LPAI subtypes H4N6 and H4N9 ever reported in poultry from Thailand.

During January to December 2009, a 12-month LBM surveillance program was carried out in LBM in Bangkok, Thailand. The LBM was visited monthly and approximately 40 samples (chicken and ducks) per market were collected at each sampling. In total, 970 samples (485 oropharyngeal and 485 cloacal swabs) were collected from 485 animals $($ chicken $=214$, ducks $=$ 271) from LBM located in the center of Bangkok. All swab samples were subjected to virus isolation by using embryonated egg inoculation according to WHO/OIE recommendations. Allantoic fluid from egg inoculation was tested for Hemagglutination (HA) titer using 1\% chicken red blood cells. Samples testing positive for HA test were subjected for influenza A virus identification and subtyping. To identify influenza A virus, real-timeRT-PCR specific for the influenza A virus matrix (M) gene was performed as previously described [10]. To subtype influenza A virus, cDNA synthesis and RT-PCR subtyping were performed. RT-PCR was performed by using primers specific for each subtype of influenza A virus $[11,12]$. In this study, 3 samples collected in June, 2009 were identified as influenza A virus subtype H4N6 $(\mathrm{n}=2)$ and H4N9 $(\mathrm{n}=1)$ while 16 samples collected in November, 2009 were identified as H10N3. On the

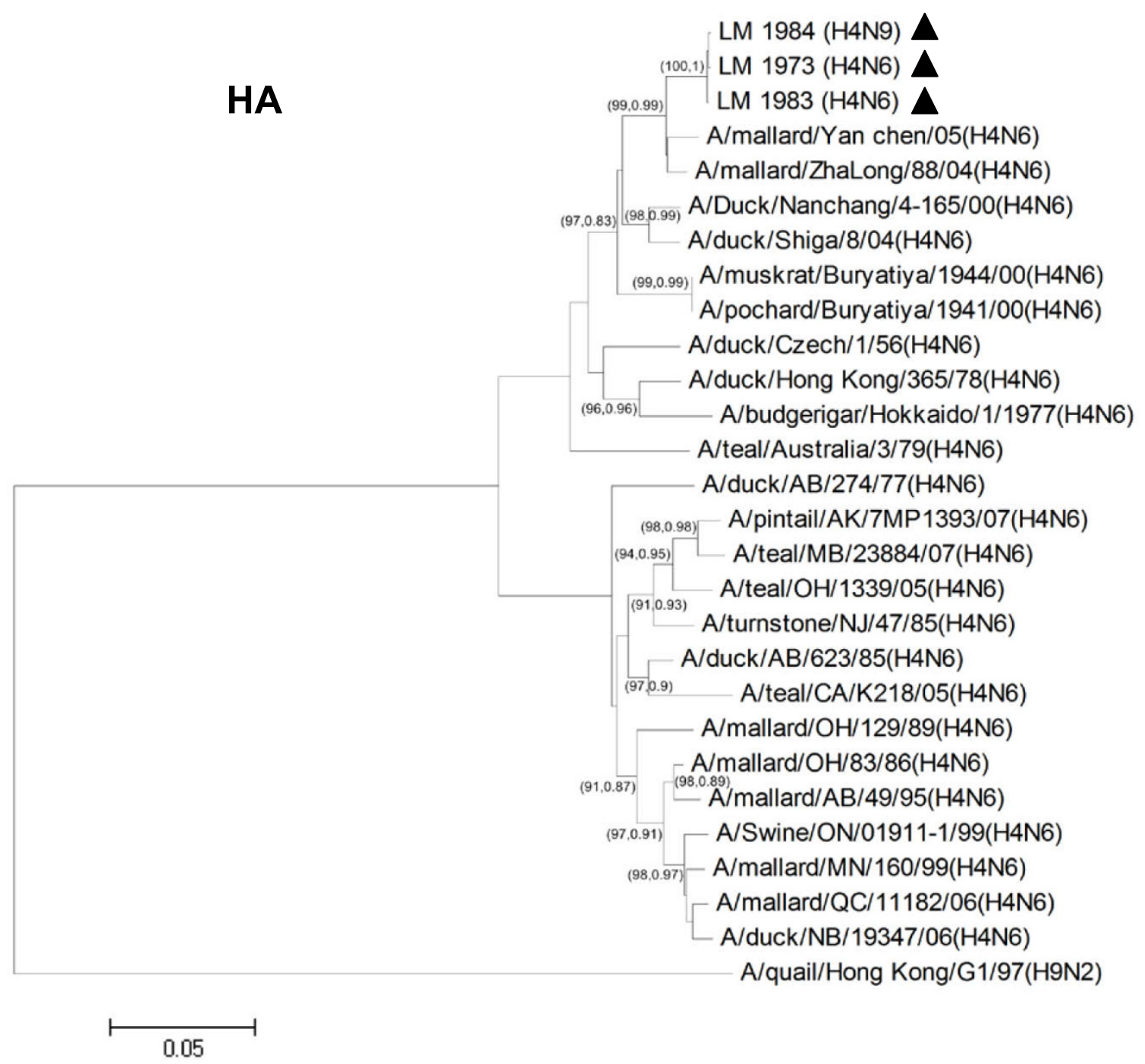

Figure 1 Phylogenetic analysis of HA gene of Thai H4N6 and H4N9 viruses and other H4N6 and H4N9 influenza A viruses. The phylogenetic tree was generated using the neighbor-joining algorithm. Bootstrap analysis with 1000 replicates and posterior probability from BMCMC analysis were performed for confirming tree topology (Bootstrap, posterior probability). The H4N6 and H4N9 influenza viruses characterized in the study are highlighted by a triangle. 
other hand, all other samples were negative for influenza A virus. Interestingly, two influenza A subtypes, H4N6 (CU-LM1983) and H4N9 (CU-LM1984), were isolated from oropharyngeal (CU-LM1983) and cloacal (CULM1984) swabs of the same duck. Both H4 subtypes have never been reported in Thailand.

After subtyping, whole genome sequencing was performed by using newly designed primers for each gene of influenza A subtypes, H4N6 and H4N9. The whole genome sequences of influenza $A$ virus subtypes H4N6 $(n=2)$ and H4N9 $(n=1)$ were submitted to the GenBank database with accession numbers as follows: A/Muscovy Duck/Thailand/CULM1973/09(H4N6) (CY062545-52), A/Muscovy Duck/Thailand/CU-LM1983/09(H4N6)(CY06255360), and A/Muscovy Duck/Thailand/CU-LM1984/09 (H4N9)(CY062561-68).
Phylogenetic analysis was performed using MEGA 4.1 program (Tempe, AZ, USA) with neighbor-joining method with Kimura 2-parameter. Nucleotide sequences of H4N6 ( $\mathrm{n}=24)$ and N9 $(\mathrm{n}=30)$ representing different geographic locations, host species and Eurasian/North American lineages were included for phylogenetic analysis. Phylogenetic analysis of the hemagglutinin (HA) gene showed that the $\mathrm{H} 4$ gene can be divided into 2 major lineages, the Eurasian and North American, respectively (Figure 1). The HA genes of both H4N6 and H4N9 viruses from Thailand were grouped into the Eurasian lineage. As for the Neuraminidase (NA) gene, phylogenetic analysis of N6 and N9 showed that both NA subtypes also clustered in the Eurasian lineage (Figure 2 and Figure 3). Phylogenetic analyses of the remaining 6 internal genes of the H4N6 and H4N9 influenza viruses were also conducted. The results

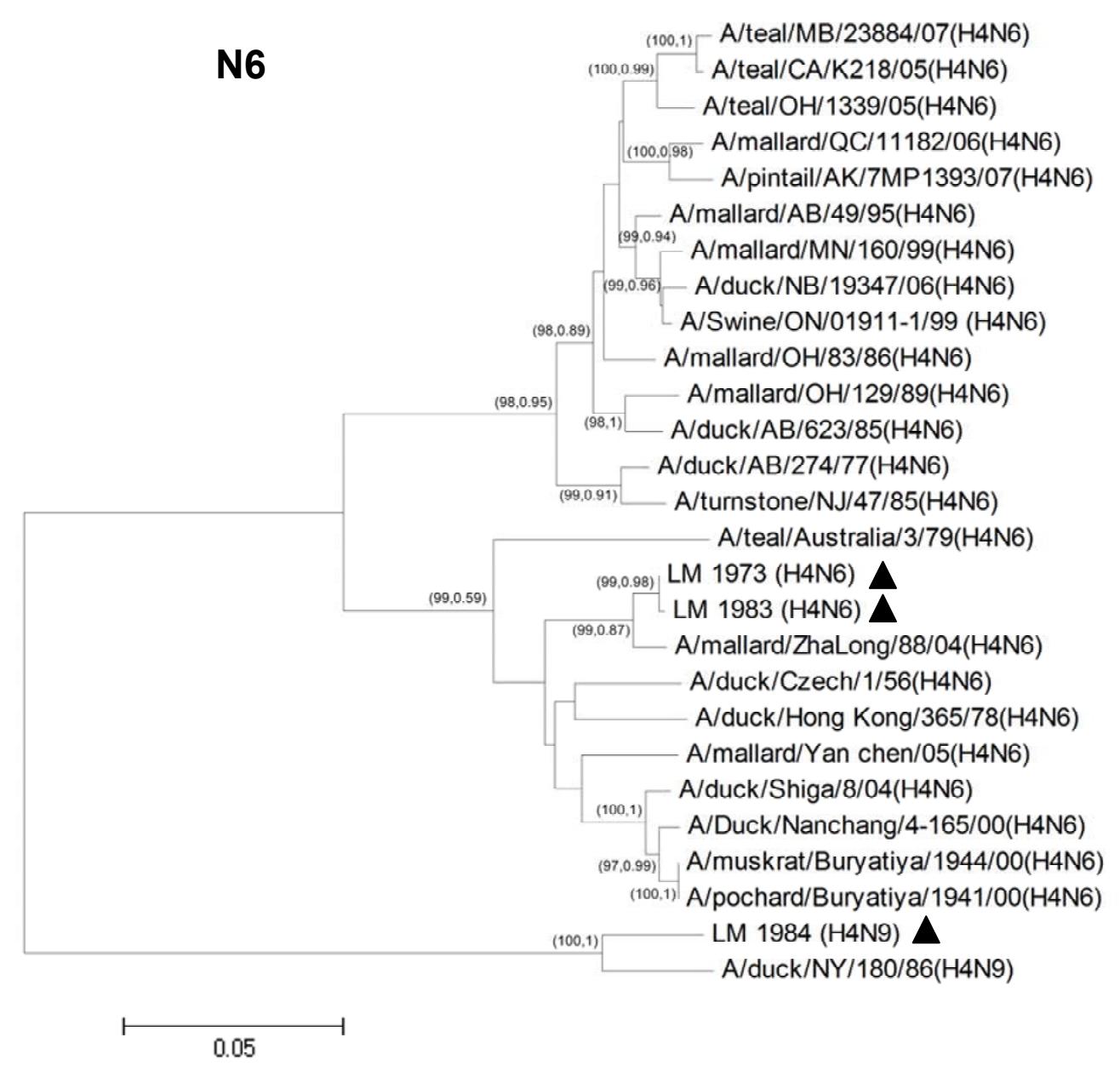

Figure 2 Phylogenetic analysis of NA gene of Thai H4N6 viruses (CU-LM1973 and CU-LM1983) and other H4N6 influenza A viruses The phylogenetic tree was generated using the neighbor-joining algorithm. Bootstrap analysis with 1000 replicates and posterior probability from BMCMC analysis were performed for confirming tree topology (Bootstrap, posterior probability). The H4N6 and influenza viruses characterized in the study are highlighted by a triangle. 


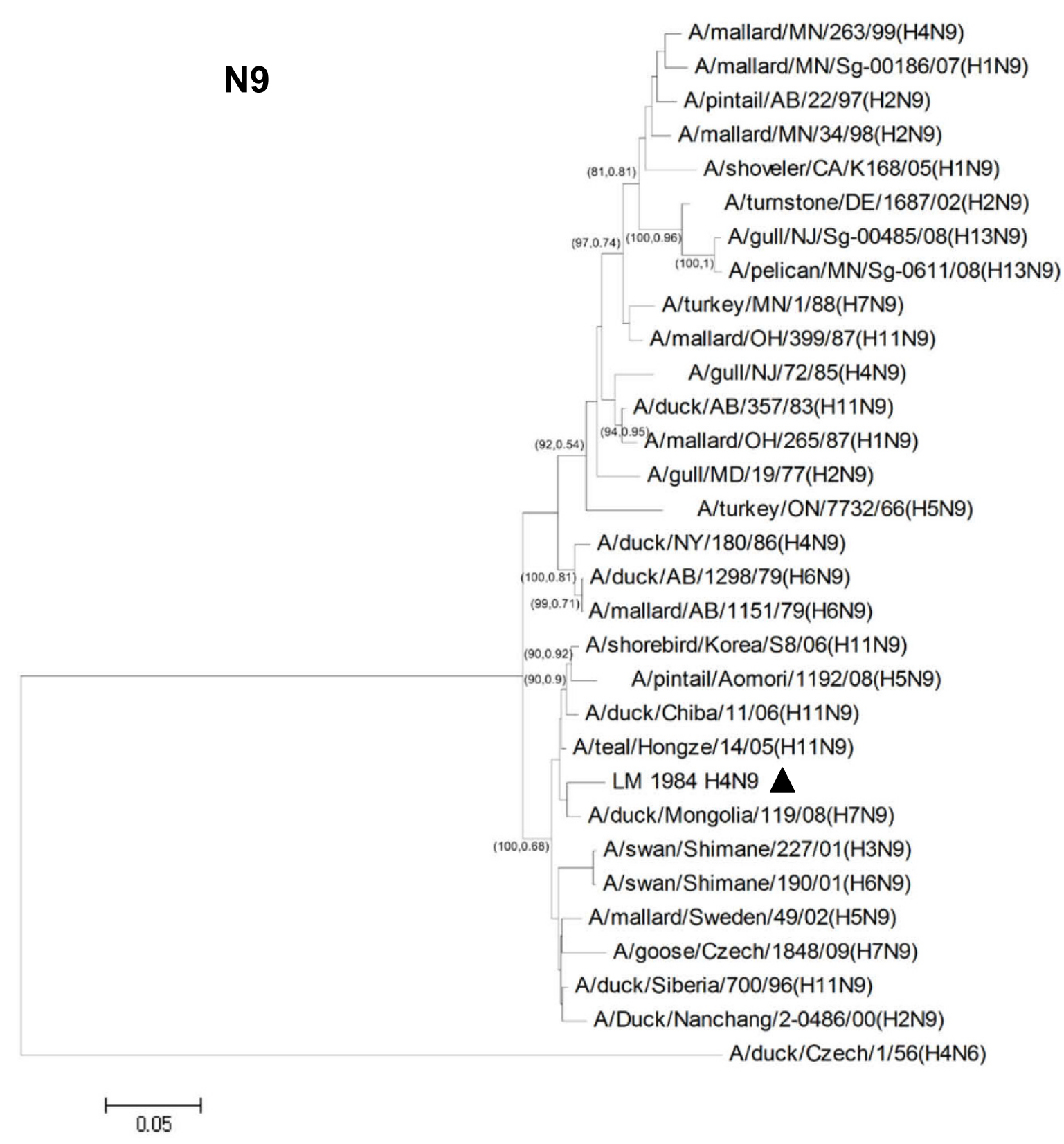

Figure 3 Phylogenetic analysis of NA gene of Thai H4N9 virus (CU-LM1984) and other N9 influenza A viruses. The phylogenetic tree was generated using the neighbor-joining algorithm. Bootstrap analysis with 1000 replicates and posterior probability from BMCMC analysis were performed for confirming tree topology (Bootstrap, posterior probability). The H4N9 influenza virus characterized in the study is highlighted by triangle.

showed that all internal genes clustered in the Eurasian lineage, similar to both HA and NA genes (Additional file 1 Fig S1). The results from phylogenetic analysis indicated that both H4N6 and H4N9 recovered from Thailand contain all gene segments derived from avian influenza A viruses of the Eurasian lineage. This may be due to the specific non-overlapping flight paths of wild and migratory birds between the Pacific and North American regions.

To genetically analyze the viruses, the nucleotide similarities of each gene of both subtypes H4N6 and H4N9 were generated by using nucleotide BLAST program available at GenBank database. Nucleotide sequences and deduced amino acids of each gene of H4N6 and 
H4N9 viruses were aligned and compared by using the MegAlign program (DNASTAR). Sequence homologies of each gene of the H4N6 and H4N9 influenza A viruses with highest nucleotide identities to reference influenza A strains are shown in Table 1 . The results showed that both H4N6 viruses (CU-LM 1973 and CU-LM 1983) and H4N9 (CU-LM 1984) display the most pronounced nucleotide identities to avian influenza viruses of the Eurasian lineage. The HA gene of all 3 viruses shows the highest nucleotide similarity to A/duck/Taiwan/ wb1104/2006(H4N6) at 97\%. The NA gene of both N6 and N9 subtypes has the highest nucleotide similarity to A/duck/Eastern China/01/2007 (H4N6) and A/duck/ Mongolia/119/2008(H7N9), respectively. H4N6 (CULM1983) and H4N9 (CU-LM1984) are highly similar in their HA gene (99.6\%) and 6 internal genes (PB2, PB1, PA, NP, NS, M) (98.4-100\%), while the NA genes of CU-LM1983(N6) and CU-LM1984(N9) show only 38.1\% nucleotide identity (64.6\% amino acid identity) because of subtype differences. This finding may suggest that co- infection with $\mathrm{H} 4 \mathrm{~N} 6$ and $\mathrm{H} 4 \mathrm{~N} 9$ occurred in the same host. It is possible that the H4N9 virus has resulted from reassortment of the $\mathrm{H} 4 \mathrm{~N} 6$ backbone and the NA gene (N9) from a separate unknown HA influenza A virus subtype (H?N9).

The deduced amino acids of the $\mathrm{H} 4$ gene of influenza A isolates were aligned and compared with those of reference H4N6 and H4N9 viruses representing the Eurasian and North American lineages published at the GenBank database using the MegAlign program. Our result showed that amino acids at the HA cleavage site in the Thai H4N6 and H4N9 viruses are "PEKASR", similar to most avian isolates in the Eurasian lineage, while those of the North American lineage including ruddy turnstone (NJ/47/85), mallard (ALB/49/95) and swine (ONT/1911-1/99) isolates are "PEKATR" (Table 2)[13] The HA cleavage site pattern of both Thai H4N6 and H4N9 indicates low pathogenic characteristics of influenza A viruses. It has been known that addition of multiple amino acids at the cleavage site such as

Table 1 Sequence homology of whole genome of three H4 isolates in this study compared to nucleotide sequences available in GenBank database

a). A/DK/THA/CU-LM1973/09 (H4N6)

\begin{tabular}{|c|c|c|c|c|}
\hline Gene & Position & Virus with the highest percentage of nucleotide identity & Genbank accession \# & $\%$ nucleotide identity \\
\hline PB2 & $1-2239$ & A/duck/Hokkaido/Nac-3/2007(H5N1) & AB355926.1 & $98 \%$ \\
\hline PB1 & $1-2271$ & A/mallard/Hokkaido/24/2009(H5N1) & AB530990.1 & $98 \%$ \\
\hline PA & $39-2133$ & A/environment/Dongting Lake/Hunan/3-9/2007(H10N8) & GQ325646.1 & $99 \%$ \\
\hline$H A$ & $1-1679$ & A/duck/Taiwan/wb1104/2006(H4N6) & GU066565.1 & $97 \%$ \\
\hline NP & $1-1462$ & A/duck/Thailand/AY-354/2008(H3N2) & FJ802402.1 & $97 \%$ \\
\hline NA & $1-1405$ & A/duck/Eastern China/01/2007(H4N6) & EU429790.1 & $97 \%$ \\
\hline M & $1-982$ & A/gull/Astrakhan/1846/1998(H13N6) & GU052229.1 & $99 \%$ \\
\hline NS & $1-817$ & A/teal/Egypt/912908/2005(H10N7) & EU599315.1 & $98 \%$ \\
\hline
\end{tabular}

b) A/Dk/THA/CU-LM1983/09 (H4N6)

\begin{tabular}{|c|c|c|c|c|}
\hline Gene & Position & Virus with the highest percentage of nucleotide identity & Genbank accession \# & $\%$ nucleotide identity \\
\hline PB2 & $1-2211$ & A/migratory duck/Hong Kong/MP206/2004(H5N2) & EF597476.1 & $97 \%$ \\
\hline PB1 & $1-2265$ & A/mallard/Hokkaido/24/2009(H5N1) & AB530990.1 & $98 \%$ \\
\hline PA & $9-2133$ & A/environment/Dongting Lake/Hunan/3-9/2007(H10N8) & GQ325646.1 & $98 \%$ \\
\hline$H A$ & $1-1682$ & A/duck/Taiwan/wb1104/2006(H4N6) & GU066565.1 & $97 \%$ \\
\hline$N P$ & $1-1462$ & A/duck/Thailand/AY-354/2008(H3N2) & FJ802402.1 & $98 \%$ \\
\hline NA & $1-1406$ & A/duck/Eastern China/01/2007(H4N6) & EU429790.1 & $97 \%$ \\
\hline M & $1-982$ & A/gull/Astrakhan/1846/1998(H13N6) & GU052229.1 & $99 \%$ \\
\hline NS & $1-821$ & A/teal/Egypt/912908/2005(H10N7) & EU599315.1 & $99 \%$ \\
\hline \multicolumn{5}{|c|}{ c) A/Dk/THA/CU-LM1984/09 (H4N9) } \\
\hline Gene & Position & Virus with the highest percentage of nucleotide identity & Genbank accession \# & $\%$ nucleotide identity \\
\hline PB2 & $55-2212$ & A/migratory duck/Hong Kong/MP206/2004(H5N2) & EF597476.1 & $97 \%$ \\
\hline PB1 & $5-2271$ & A/mallard/Hokkaido/24/2009(H5N1) & AB530990.1 & $98 \%$ \\
\hline PA & $9-2133$ & A/environment/Dongting Lake/Hunan/3-9/2007(H10N8) & GQ325646.1 & $99 \%$ \\
\hline$H A$ & $1-1684$ & A/duck/Taiwan/wb1104/2006(H4N6) & GU066565.1 & $97 \%$ \\
\hline NP & $1-1470$ & A/duck/Thailand/AY-354/2008(H3N2) & FJ802402.1 & $98 \%$ \\
\hline NA & 24-1377 & A/duck/Mongolia/119/2008(H7N9) & AB481213.1 & $97 \%$ \\
\hline M & $1-955$ & A/gull/Astrakhan/1846/1998(H13N6) & GU052229.1 & $99 \%$ \\
\hline NS & $1-818$ & A/teal/Egypt/912908/2005(H10N7) & EU599315.1 & $99 \%$ \\
\hline
\end{tabular}


Table 2 Genetic analysis of amino acids at HA cleavage site and receptor binding sites of $\mathrm{H} 4 \mathrm{gene}$ of three $\mathrm{H} 4$ isolates in this study and reference H4N6 and H4N9 virus

\begin{tabular}{|c|c|c|c|c|c|c|c|c|}
\hline \multirow[b]{2}{*}{ H3 numbering system* } & \multirow{2}{*}{$\begin{array}{c}\text { HA cleavage } \\
320-329\end{array}$} & \multicolumn{7}{|c|}{ Receptor-binding site } \\
\hline & & 98 & 153 & 155 & 183 & 190 & 194 & 195 \\
\hline H4 position & $338-343$ & 110 & 165 & 167 & 196 & 203 & 207 & 208 \\
\hline A/Dk/TH/CU-LM1973/09 (H4N6) & PEKASR & Y & W & V & $\mathrm{H}$ & $\mathrm{E}$ & L & Y \\
\hline A/Dk/TH/CU-LM1983/09 (H4N6) & PEKASR & Y & W & V & $\mathrm{H}$ & E & L & Y \\
\hline A/DK/TH/CU-LM1984/09 (H4N9) & PEKASR & Y & W & V & $\mathrm{H}$ & E & L & Y \\
\hline A/Dk/Czech/1/56 (H4N6) & PEKASR & Y & W & V & $\mathrm{H}$ & E & L & Y \\
\hline A/Muskrat/Buryatiya/1944/00 (H4N6) & PEKAPR & Y & W & V & $\mathrm{H}$ & E & L & Y \\
\hline A/mallard/Yan Chen/2005 (H4N6 & PEKASR & Y & W & V & $\mathrm{H}$ & $\mathrm{E}$ & L & Y \\
\hline A/turnstone/NJ/47/85 (H4N6) & PEKATR & Y & W & $\mathrm{T}$ & $\mathrm{H}$ & $\mathrm{E}$ & L & Y \\
\hline A/mallard/Alberta/49/1995(H4N6) & PEKATR & Y & W & $\mathrm{T}$ & $\mathrm{H}$ & $\mathrm{E}$ & L & Y \\
\hline A/Sw/ON/01911-1/99 (H4N6) & PEKATR & Y & W & $\mathrm{T}$ & $\mathrm{H}$ & $\mathrm{E}$ & L & Y \\
\hline A/Dk/NY/180/86 (H4N9) & PEKATR & Y & W & $\mathrm{T}$ & $\mathrm{H}$ & E & L & Y \\
\hline
\end{tabular}

\begin{tabular}{lcccccc} 
& Left edge of receptor-binding site & & \\
\cline { 2 - 6 } H3 numbering system & 224 & 225 & 226 & 227 & 228 & 229 \\
H4 position & 237 & 238 & 239 & 240 & 241 & 242 \\
\hline A/Dk/TH/CU-LM1973/09 (H4N6) & $\mathrm{R}$ & $\mathrm{G}$ & $\mathrm{Q}$ & $\mathrm{S}$ & $\mathrm{G}$ & $\mathrm{R}$ \\
A/Dk/TH/CU-LM1983/09 (H4N6) & $\mathrm{R}$ & $\mathrm{G}$ & $\mathrm{Q}$ & $\mathrm{S}$ & $\mathrm{G}$ & $\mathrm{R}$ \\
A/Dk/TH/CU-LM1984/09 (H4N9) & $\mathrm{R}$ & $\mathrm{G}$ & $\mathrm{Q}$ & $\mathrm{S}$ & $\mathrm{G}$ & $\mathrm{R}$ \\
A/Dk/Czech/1/56 (H4N6) & $\mathrm{R}$ & $\mathrm{G}$ & $\mathrm{Q}$ & $\mathrm{S}$ & $\mathrm{G}$ & $\mathrm{R}$ \\
A/Muskrat/Buryatiya/1944/00 (H4N6) & $\mathrm{R}$ & $\mathrm{G}$ & $\mathrm{Q}$ & $\mathrm{S}$ & $\mathrm{G}$ & $\mathrm{R}$ \\
A/mallard/Yan Chen/2005 (H4N6 & $\mathrm{R}$ & $\mathrm{G}$ & $\mathrm{Q}$ & $\mathrm{S}$ & $\mathrm{G}$ & $\mathrm{R}$ \\
A/turnstone/NJ/47/85 (H4N6) & $\mathrm{R}$ & $\mathrm{G}$ & $\mathrm{Q}$ & $\mathrm{S}$ & $\mathrm{G}$ & $\mathrm{R}$ \\
A/mallard/Alberta/49/1995(H4N6) & $\mathrm{R}$ & $\mathrm{G}$ & $\mathrm{Q}$ & $\mathrm{S}$ & $\mathrm{G}$ & $\mathrm{R}$ \\
A/Sw/ON/01911-1/99 (H4N6) & $\mathrm{R}$ & $\mathrm{G}$ & $\mathrm{L}$ & $\mathrm{S}$ & $\mathrm{S}$ & $\mathrm{R}$ \\
A/Dk/NY/180/86 (H4N9) & $\mathrm{R}$ & $\mathrm{G}$ & $\mathrm{Q}$ & $\mathrm{S}$ & $\mathrm{G}$ & $\mathrm{R}$
\end{tabular}

\begin{tabular}{l} 
H3 numbering system \\
H4 position \\
\hline A/Dk/TH/CU-LM1973/09 (H4N6) \\
A/Dk/TH/CU-LM1983/09 (H4N6) \\
A/Dk/TH/CU-LM1984/09 (H4N9) \\
A/Dk/Czech/1/56 (H4N6) \\
A/Muskrat/Buryatiya/1944/00 (H4N6) \\
A/mallard/Yan Chen/2005 (H4N6 \\
A/turnstone/NJ/47/85 (H4N6) \\
A/mallard/Alberta/49/1995(H4N6) \\
A/Sw/ON/01911-1/99 (H4N6) \\
A/Dk/NY/180/86 (H4N9)
\end{tabular}

Right edge of receptor-binding site

\begin{tabular}{ccccc}
\hline 134 & 135 & 136 & 137 & 138 \\
146 & 147 & 148 & 149 & 150 \\
\hline$G$ & K & S & G & A \\
G & K & S & G & A \\
G & K & S & G & A \\
G & K & S & G & A \\
G & K & S & G & A \\
G & K & S & G & A \\
G & K & S & G & A \\
G & K & S & G & A \\
G & K & S & G & A \\
G & K & S & G & A
\end{tabular}

Arginine (R) and Lysine (K) may turn LPAI into HPAI [14]. However, to further confirm virus pathogenicity, an intravenous pathogenicity index test (IVPI) should be performed [15]. Amino acids at receptor binding sites (HA98,153,155,183,190, 194,195 (H3 numbering system)) were also analyzed (Table 2). In this study, the amino acids of Thai H4N6 and H4N9 at positions 224-229 and 134-138 were "RGQSGR" and "GKSGA", respectively. It is noteworthy that the receptor binding sites of H4N6 and H4N9 especially, Q226 and G228 are similar to all H4N6 in both Eurasian and North American lineages suggesting that these viruses would preferentially bind to 2,3 linked sialic acid receptors predominant in avian species [16]. One H4N6 virus from swine (A/Swine/Ontario/01911-1/99) contains amino acid changes at positions $226(\mathrm{Q} 226 \mathrm{~L})$ and 228 
(G228S) indicating high affinity of the virus to SA $2,6 \mathrm{Gal}$ receptors which are dominant in mammalian species $[13,16]$. N-linked glycosylation sites of the $\mathrm{H} 4$ gene were also analyzed. Three $\mathrm{N}$-linked glycosylation sites in HA1 and one glycosylation site in HA2 were observed in Thai H4 viruses (Figure 4). It should be noted that one glycosylation site in HA1 of Thai H4 influenza $A$ isolates was absent due to the amino acid changes N2D resulting in loss of glycosylation ability [17]. Thai H4N6 and H4N9 contain less N-linked glycosylation sites than the viruses described in a previous report [17]. Previous studies reported that an absent of $\mathrm{N}$-liked glycosylation site of HA protein would altered biological functions of the virus [18,19].

The influenza A virus subtypes H4N6 and H4N9 found in LBMs can be a potential risk to birds and humans. The viruses were shed in feces and contaminated the wire stack cages in LBM. This setting provides an appropriate condition for virus transmission among birds kept in the cage nearby or below [20]. Consequently, a small outbreak of influenza A viruses H4N6 and H4N9 in the birds in LBM can occur. Fortunately, the H4N6 and H4N9 viruses circulating in this LBM are of low virulence and hardly capable of infecting humans. However, if HPAI or newly reassorted viruses with high virulence circulate in the markets in the future, this would pose a threat to vendors, consumers and people who enter the markets. For example, in China, HPAI H5N1 infection was reported in one patient who had visited LBMs. The study showed that LBM can be a potential source of HPAI in a local community and infection of humans can occur not only by direct but also by indirect contact [4].

The public should be made aware of the significance of the influenza viruses, the hosts, the environment and potential virus reassortment especially in LBMs. Examples of influenza prevention and control strategies in the LBM include 1) creating a rest day to interrupt the LBM's flow system, which could significantly reduce the occurrence of the virus in LBMs [21], 2) disinfection of fomites in LBMs as the presence of influenza A virus in fomites has been documented [22], 3) educational campaigns, vendors should be informed on how to protect themselves from influenza A virus infection. Since surveillance of influenza A virus in LBMs can serve as a good early warning system [6], influenza A viruses isolated in LBMs can reflect the status and the diversity of the viruses circulating in local communities. Continuous influenza A virus surveillance in LBMs should be performed in order to understand the epidemiology of influenza A virus in LBMs and to monitor influenza virus evolution.

In conclusion, influenza A virus subtypes H4N6 and H4N9 were isolated from ducks in a live bird market in Bangkok, Thailand. Both H4N6 and H4N9 subtypes

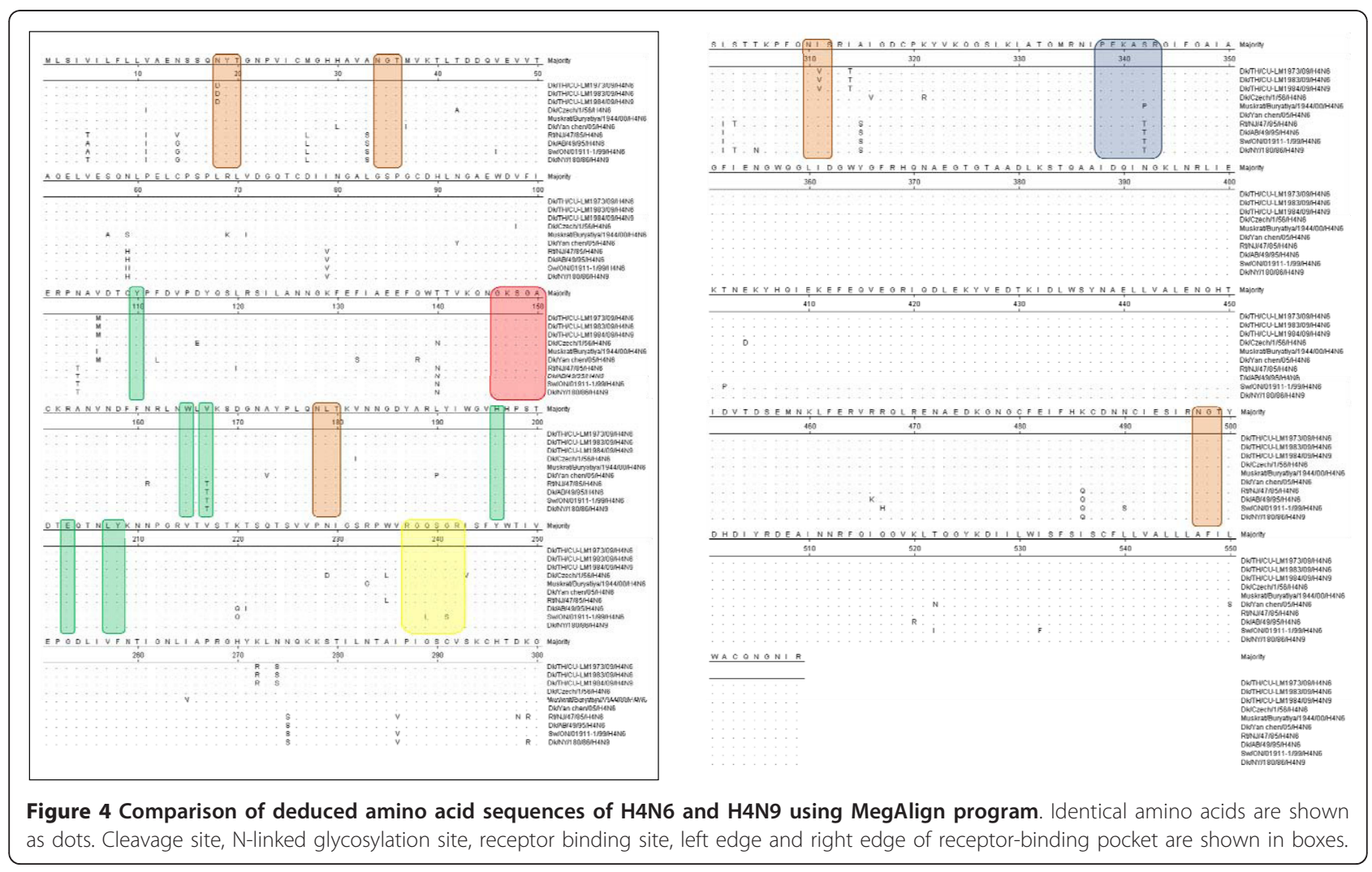


recovered from ducks had never been reported in Thailand. Whole genome sequences of the viruses were determined and submitted to the respective databases. Phylogenetic analysis of $\mathrm{H} 4 \mathrm{~N} 6$ and $\mathrm{H} 4 \mathrm{~N} 9$ viruses showed that gene segments of the viruses were derived from the Eurasian linage. Genetic analysis of the viruses also showed that the viruses display low pathogenic characteristics, with amino acids specific to avian influenza viruses.

\section{Additional material}

Additional file 1: Figure S1. Phylogenetic analysis of internal genes of Thai $\mathrm{H} 4 \mathrm{~N} 6$ and $\mathrm{H} 4 \mathrm{~N} 9$ viruses and other H4N6 and H4N9 influenza A viruses. The phylogenetic tree was generated using the neighbor-joining algorithm. Bootstrap analysis with 1000 replicates and posterior probability from BMCMC analysis were performed for confirming tree topology (Bootstrap, posterior probability). The H4N6 and H4N9 influenza viruses characterized in the study are present in triangle.

\section{Acknowledgements}

This work has been funded in whole with federal funds from the National Institute of Allergy and Infectious Disease, National Institute of Health, Department of Health and Human Services, under Contract No. HHSN266200700007c. Its contents are solely the responsibility of the authors and do not necessarily represent the official views of the $\mathrm{NIH}$. We also would like to thank the National Research Council of Thailand (NRCT, Thailand) and the National Research University of CHE and the Ratchadaphiseksomphot Endowment Fund (HR1155A) for grant support to AA.

We would like to thank Chulalongkorn University for its financial support in the Emerging and Re-emerging Infectious Diseases in Animals, Research Unit. We would like to thank Ms. Petra Hirsch for reviewing the manuscript.

\section{Author details}

${ }^{1}$ Emerging and Re-emerging Infectious Diseases in Animals, Research Unit, Faculty of Veterinary Science, Chulalongkorn University, Bangkok, Thailand. ${ }^{2}$ Department of Veterinary Public Health, Faculty of Veterinary Science, Chulalongkorn University, Bangkok, Thailand. ${ }^{3}$ Department of Pathology, Faculty of Veterinary Science, Chulalongkorn University, Bangkok, Thailand.

\section{Authors' contributions}

TW conducted sample collection, virus isolation, virus identification, genome sequencing, phylogenetic analysis and drafted the manuscript. MW, SB, NB participated in sample collection, virus isolation and whole genome sequencing. PK helped designed LBM surveillance, data analyses. AA designed LBM surveillance, data analyses and final approval of the manuscript. All authors read and approved the final manuscript.

\section{Competing interests}

The authors declare that they have no competing interests.

Received: 21 January 2011 Accepted: 21 March 2011

Published: 21 March 2011

\section{References}

1. Nguyen DC, Uyeki TM, Jadhao S, Maines T, Shaw M, Matsuoka Y, Smith C, Rowe T, Lu X, Hall H, et al: Isolation and characterization of avian influenza viruses, including highly pathogenic $\mathrm{H} 5 \mathrm{~N} 1$, from poultry in live bird markets in Hanoi, Vietnam, in 2001. J Virol 2005, 79:4201-4212.

2. Kim JA, Cho SH, Kim HS, Seo SH: H9N2 influenza viruses isolated from poultry in Korean live bird markets continuously evolve and cause the severe clinical signs in layers. Vet Microbiol 2006, 118:169-176.
3. Chan PK: A review on human influenza A H5N1 infections in Hong Kong Sci China C Life Sci 2009, 52:412-418.

4. Wang $M$, Di B, Zhou DH, Zheng BJ, Jing H, Lin YP, Liu YF, Wu XW, Qin PZ, Wang $Y L$, et al: Food markets with live birds as source of avian influenza. Emerg Infect Dis 2006, 12:1773-1775.

5. Cardona C, Yee K, Carpenter T: Are live bird markets reservoirs of avian influenza? Poult Sci 2009, 88:856-859.

6. Webster RG: Wet markets-a continuing source of severe acute respiratory syndrome and influenza? Lancet 2004, 363:234-236.

7. Liu M, He S, Walker D, Zhou N, Perez DR, Mo B, Li F, Huang X, Webster RG, Webby RJ: The influenza virus gene pool in a poultry market in South central china. Virology 2003, 305:267-275.

8. Suarez DL, Senne DA: Sequence analysis of related low-pathogenic and highly pathogenic H5N2 avian influenza isolates from United States live bird markets and poultry farms from 1983 to 1989 . Avian Dis 2000, 44:356-364.

9. Amonsin A, Choatrakol C, Lapkuntod J, Tantilertcharoen R, Thanawongnuwech R, Suradhat S, Suwannakarn K, Theamboonlers A, Poovorawan Y: Influenza virus (H5N1) in live bird markets and food markets, Thailand. Emerg Infect Dis 2008, 14:1739-1742.

10. Payungporn $S$, Phakdeewirot $P$, Chutinimitkul $S$, Theamboonlers $A$, Keawcharoen J, Oraveerakul K, Amonsin A, Poovorawan Y: Single-step multiplex reverse transcription-polymerase chain reaction (RT-PCR) for influenza A virus subtype H5N1 detection. Viral Immunol 2004, 17:588-593.

11. Tsukamoto K, Ashizawa H, Nakanishi K, Kaji N, Suzuki K, Okamatsu M, Yamaguchi S, Mase M: Subtyping of avian influenza viruses $\mathrm{H} 1$ to $\mathrm{H} 15$ on the basis of hemagglutinin genes by PCR assay and molecular determination of pathogenic potential. J Clin Microbiol 2008, 46:3048-3055.

12. Tsukamoto K, Ashizawa T, Nakanishi K, Kaji N, Suzuki K, Shishido M, Okamatsu M, Mase M: Use of reverse transcriptase PCR to subtype N1 to N9 neuraminidase genes of avian influenza viruses. J Clin Microbiol 2009, 47:2301-2303.

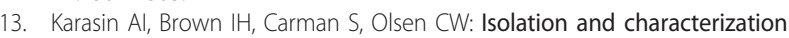
of H4N6 avian influenza viruses from pigs with pneumonia in Canada. J Virol 2000, 74:9322-9327.

14. Horimoto T, Ito T, Alexander DJ, Kawaoka Y: Cleavability of hemagglutinin from an extremely virulent strain of avian influenza virus containing a unique cleavage site sequence. J Vet Med Sci 1995, 57:927-930.

15. Wood GW, Banks J, Strong I, Parsons G, Alexander DJ: An avian influenza virus of $\mathrm{H} 10$ subtype that is highly pathogenic for chickens, but lacks multiple basic amino acids at the haemagglutinin cleavage site. Avian Pathol 1996, 25:799-806.

16. Bateman AC, Busch MG, Karasin Al, Bovin N, Olsen CW: Amino acid 226 in the hemagglutinin of H4N6 influenza virus determines binding affinity for alpha2,6-linked sialic acid and infectivity levels in primary swine and human respiratory epithelial cells. J Virol 2008, 82:8204-8209.

17. Donis RO, Bean WJ, Kawaoka Y, Webster RG: Distinct lineages of influenza virus $\mathrm{H} 4$ hemagglutinin genes in different regions of the world. Virology 1989, 169:408-417.

18. Matrosovich M, Zhou N, Kawaoka Y, Webster R: The surface glycoproteins of $\mathrm{H} 5$ influenza viruses isolated from humans, chickens, and wild aquatic birds have distinguishable properties. J Virol 1999, 73:1146-1155.

19. Roberts PC, Garten W, Klenk HD: Role of conserved glycosylation sites in maturation and transport of influenza A virus hemagglutinin. J Virol 1993, 67:3048-3060.

20. Yee KS, Carpenter TE, Farver TB, Cardona CJ: An evaluation of transmission routes for low pathogenicity avian influenza virus among chickens sold in live bird markets. Virology 2009, 394:19-27.

21. Lau EH, Leung YH, Zhang LJ, Cowling BJ, Mak SP, Guan Y, Leung GM, Peiris JS: Effect of interventions on influenza A (H9N2) isolation in Hong Kong's live poultry markets, 1999-2005. Emerg Infect Dis 2007, 13:1340-1347.

22. Boone $S A$, Gerba CP: The occurrence of influenza $A$ virus on household and day care center fomites. J Infect 2005, 51:103-109.

doi:10.1186/1743-422X-8-131

Cite this article as: Wisedchanwet et al:: Genetic characterization of avian influenza subtype H4N6 and H4N9 from live bird market, Thailand. Virology Journal 2011 8:131. 